\title{
De dinámicas barriales, muertes violentas y registro estadístico: una aproximación etnográfica
}

\author{
As dinâmicas dos bairros, mortes violentas e registro estatístico: uma \\ abordagem etnográfica
}

Of neighborhood dynamics, violent deaths and statistical registration: an
ethnographic inquiry

Ma. Elea Maglia ${ }^{1}$

Lic. Martín Di Marco²

Lic. Marina García Acevedo 3

\begin{abstract}
Resumen
Este artículo se propone analizar los procesos sociales, morales y relacionales que condicionan el registro de datos estadísticos sobre muertes violentas, a partir del enfoque etnográfico. Se parte del presupuesto de que si bien la tasa de homicidio es empleada como uno de los indicadores más sólidos a partir del cual analizar los niveles de violencia y crimen en una sociedad, no está eximida de condicionantes sociales. Los procesos por medio de los cuales se registran y clasifican las muertes violentas están atravesados por dinámicas sociales, determinadas por los contextos locales y las diversas creencias y prácticas de los actores. Con el objetivo de desandar las lógicas que hacen posible el subregistro y omisión de homicidios, se realizó una etnografía en 2016 en un barrio caracterizado por la vulnerabilidad social y altos niveles de violencia, ubicado al suroeste de la Ciudad Autónoma de Buenos Aires, en el Conurbano Bonaerense. La metodología incluyó la realización de observaciones participantes en diferentes espacios del barrio, entrevistas abiertas, semi-estructuradas y grupales. En función de ello, a partir del análisis específico de un caso en el que no se registró la muerte violenta de un joven y otro en la que se clasificaron 2 homicidios como suicidios, se desandan las lógicas barriales que hicieron posible este tratamiento de la muerte violenta y se reflexiona sobre dinámicas sociales producidas y reproducidas al interior del barrio.
\end{abstract}

Palavras claves: Etnografía; Muertes violentas; Dinámicas barriales; Subregistro.

\section{Resumo}

Este artigo tem como objetivo analisar os processos sociais, morais e relacionais que condicionam o registro de dados estatísticos sobre mortes violentas, com base na abordagem etnográfica. Baseia-se no pressuposto de que, embora a taxa de homicídios seja utilizada como um dos indicadores mais sólidos para analisar os níveis de violência e crime em uma sociedade, não está isento de condicionantes sociais. Os processos pelos quais as mortes violentas são registradas e classificadas são atravessados pelas dinâmicas sociais, determinadas pelos

${ }^{1}$ Licenciada en Sopciología (UBA), Magíster en Antropologia Social (FLACSO) y Doctoranda en Antropología Social (IDAES/UNSAM), Beca Doctoral CONICET; Universidad Nacional de San Martin; San Martin, Argentina; magliaelea@gmail.com.

${ }^{2}$ Licenciado en Sociología (UBA), Becario Doctoral de CONICET/IIGG; Universidad de Buenos Aires; Buenos Aires, Argentina; mh.dimarco@gmail.com.

${ }^{3}$ Licenciada en Sociología (UBA), Maestranda en Administración y Políticas Públicas (Universidad de San Andrés); Centro de Estudios Legales y Sociales (CELS); marina6arcia@ hotmail.com. 
contextos locais e pelas diversas crenças e práticas dos atores. Com o objetivo de elucidar as lógicas que possibilitam o sub-registro e a omissão de homicídios, uma etnografia foi realizada em 2016 em um bairro caracterizado por vulnerabilidade social e altos níveis de violência, localizado a sudoeste da Cidade Autônoma de Buenos Aires, na Conurbação Bonaerense. A metodologia incluiu a realização de observações participantes em diferentes áreas do bairro, entrevistas abertas, semi-estruturadas e em grupo. Com base nisso, a partir da análise específica de um caso em que a morte violenta de um jovem não foi registrada e outra em que dois homicídios foram classificados como suicídios, as lógicas do bairro que tornaram possível este tratamento da morte violência são descritas e a dinâmica social produzida e reproduzida dentro do bairro é analisada.

Palavras-chave: Etnografia; Mortes violentas; Dinâmica do bairro; Sub-registro.

\begin{abstract}
This paper analyses social, moral and relational processes which condition the gathering and registration of statistical data related to violent deaths, from an ethnographical approach. The premise in this paper is that while the homicide rate is one of the most solid indicators to analyze violence and crime levels in society, it is not excluded from social conditionings. The gathering, recording and classification processes of violent deaths are shaped by social dynamics determined by local contexts and the wide range of beliefs and practices of actors. With the purpose of elucidating the reasons which make possible the underregistration and lack of registration of homicides, an ethnographical research study was carried out during 2016 in a socially-vulnerable neighborhood with high rates of violence, located Southwest of Buenos Aires Capital City, in Buenos Aires Conurbation. The methodology consisted of participant observations in several areas of the neighborhood, as well as unstructured, semi-structured and group interviews. Based on the analysis of a case where the violent death of a young boy was not registered and another case where 2 homicides was classified as suicides, neighborhood's dynamics are discussed as conditioning factors of how these deaths are treated and classified, and the social dynamics produced and reproduced within the neighborhood are analyzed.
\end{abstract}

Keywords: Ethnography; Violent deaths, Neighbourhood dynamics; Underregistrarion.

\title{
1. Introducción
}

En el marco de los estudios sociológicos y antropológicos de la violencia, este artículo parte del interrogante sobre qué procesos sociales y morales condicionan el registro de datos sobre muertes violentas. A partir de un trabajo de campo etnográfico en el barrio "Santa Ana" del Conurbano Bonaerense (Argentina), el objetivo de este artículo es describir y dilucidar los procesos sociales, morales y relacionales que condicionan el registro de datos estadísticos sobre muertes violentas. Este objetivo se vincula con un propósito analítico más general que es reflexionar sobre la tasa de homicidio entendida como un fenómeno social en torno al que circulan significados, prácticas, moralidades y formas de ver el mundo.

En los estudios de ciencias sociales, la tasa de homicidio es empleada como el indicador más sólido a partir del cual analizar los niveles de violencia y crimen de una sociedad (AZEVEDO y CIFALI, 2016; BRICEÑO-LEÓN, 2012). Ello se debe tanto a su mayor nivel de denuncia frente a la "cifra negra" presente en otro tipo de delitos, como robos, hurtos y delitos contra la integridad sexual (MIGUEZ e ISLA, 2010); así como a la visibilidad que adquieren los decesos dada la serie de procesos burocráticos que se desencadenan tras la 
muerte de una persona. Los datos sobre homicidios suelen ser procesados a partir de registros recabados de personas denunciantes y de documentos administrativos producidos en centros de salud, dependencias policiales y el poder judicial.

El subregistro de las muertes violentas ha sido discutido en escenarios específicos, por ejemplo en contextos de conflictos bélicos, guerras civiles o casos de fuerte inestabilidad social e institucional, en los que, si bien los niveles de homicidio son altos, la tasa de denuncias es baja, como los casos mexicano, colombiano, venezolano, y salvadoreño (BUVINIC; MORRISON, 1999; DAMMERT; ZÚÑIGA, 2007; DÁVILA-CERVANTES; PARDO-MONTAÑO; 2014, MEXICO EVALÚA, 2011, 2017). No obstante, la calidad de los datos estadísticos de muertes violentas en situaciones no-excepcionales también ha sido objeto de investigación de diferentes disciplinas, que han destacado los escollos metodológicos -vinculados a cómo registrar los datos operativamente- y políticoinstitucionales relacionados con la infraestructura de organismos y actores en torno al tema (GOVEA BASCH, 2010; MAZZEO, 2005; BALL; REED, 2016).

Puntualmente en el contexto argentino, se ha destacado como problema estatal y científico (MAZZEO, 2005; SPINELLI, ALAZRAQUI y MACÍAS, 2005; YUNE y ZUBAREW, 1999) el gran volumen de muertes violentas indeterminadas: aquellas clasificadas como debidas a causas externas o violentas (término proveniente de la Clasificación Internacional de Enfermedades o CIE), pero no discriminadas entre homicidio ${ }^{4}$, suicidio o accidente. Esta dificultad ha sido subrayada en los análisis que utilizan como fuente las bases de datos del ministerio de salud (FLEITAS, 2014), en aquellas investigaciones que profundizan en casos de presuntos suicidios y descubren que encubren homicidios, muchas veces femicidios (FERNÁNDEZ, 2012), en estudios sobre muertes de personas en conflicto con la ley penal, donde existe un nivel de responsabilidad estatal (PROCUVIN, s/f). Sin embargo, pocos estudios indagan en las lógicas sociales implicadas en el registro de estas muertes.

En la omisión, subregistro e indeterminación de las muertes por causas violentas intervienen procesos sociales que convierten a estos fenómenos en un objeto de estudio de difícil "acceso" desde las estrategias metodológicas cuantitativas (MALDONADO ARANA, 2013; SCHEPER-HUGHES, 2010). Por este motivo, las aproximaciones cualitativas han sido fértiles en producir datos ricos y, asimismo, en proponer hipótesis que permitan entender los

\footnotetext{
${ }^{4} \mathrm{Al}$ hacer referencia a homicidio, haremos exclusiva referencia a homicidio doloso
} 
condicionantes sociales que intervienen en el registro de datos (ALONSO, 1986; OTERO, 2006; RAMÍREZ HITA, 2009).

En el marco de estas discusiones, la propuesta de este artículo es reflexionar sobre los procesos sociales y morales que condicionan el registro de datos sobre muertes violentas a partir del método etnográfico. Siguiendo la línea de investigaciones latinoamericanas sobre violencias, barrios populares y Estado (AUYERO y BERTI, 2013; BERMÚDEZ, 2011; MENÉNDEZ, 2012; ÁLVAREZ, 2004), se explorarán etnográficamente los procesos relacionales y simbólicos que atraviesan el subregistro y omisión de homicidios, en el barrio que llamaremos "Santa Ana". Esta perspectiva metodológica permite desandar dinámicas locales asociadas a los valores, moralidades y prácticas que, en determinadas circunstancias, se activan a la hora de registrar y catalogar las muertes violentas. Rescataremos la dimensión procesual que implica el registro en fuentes oficiales de muertes violentas, a partir del análisis de las lógicas desatadas tras algunas de ellas en este barrio, puntualmente de la reflexión sobre los factores micro-sociales que incidieron en los tratamientos, discursos y creencias en torno al registro de estas muertes.

En esta investigación se partió de la premisa de que la producción de conocimiento es una relación social, lo que implica la puesta en juego de la reflexividad del investigador y de los sujetos que desea conocer; es decir, la conjunción de experiencias, expectativas, emociones, encuentros y desencuentros entre estos actores (GUBER, 2005). El trabajo de campo etnográfico se orientó a comprender los fenómenos sociales que se desencadenan tras una muerte, desde la perspectiva de los actores. La investigación consistió en la realización de observaciones participantes en diferentes espacios del barrio (una comisaría, plazas, un centro de salud, una escuela, "esquinas"), así como de entrevistas abiertas, semi-estructuradas y grupales a jóvenes —algunos de ellos incluidos en programas sociales-, familiares de víctimas de violencia y homicidio, policías, docentes, médicos de la salita, entre otros.

El artículo se organiza en cinco apartados: el primero sistematiza los textos que auspician de marco teórico y antecedentes en el tema; el segundo describe cuantitativamente las principales características de la Provincia de Buenos Aires y cualitativamente al barrio Santa Ana; en el tercero son desarrolladas dos escenas a partir de las cuales se dará cuenta de las lógicas barriales y formas de registrar las muertes violentas; en el cuarto apartado se reflexiona sobre cómo estos muertos expresan la fragmentación social en el barrio; el quinto y último apartado reflexiona sobre la muerte y los procesos de victimización a ella o no asociados. 


\section{Puntos de partida}

Si bien sabemos que las prácticas sociales, incluyendo las burocráticas, no se agotan en las prescripciones formales o en el "deber ser", sino que son reguladas en función de clivajes sociales y procesos relacionales (MATUS, 1992; GALVANI I., 2014; BOURDIEU, 1979), esta premisa general pareciera quedar relegada a la hora de analizar cómo se registran los homicidios. No hay que perder de vista que los documentos producidos por las agencias judiciales y sanitarias materializan relaciones de poder, sedimentan juegos estratégicos, de acción y reacción, de dominación y retracción, de lucha (FOUCAULT, 1996). De aquí que amerite la pregunta: ¿Existe algún tipo de situación en la que se ponga en tensión el deber de registrar las muertes violentas por parte los funcionarios con otras lógicas de acción? ¿Qué lugar ocupan la omisión o los registros incompletos?

Por otra parte, existe cierto consenso en las investigaciones en torno a la muerte respecto a que el cuerpo muerto trasciende el acto de morir, porque sobre él se desarrollan procesos simbólicos y acciones socialmente significativas, volviéndose con ello un "cuerpo significante" (PANIZO, 2011). Partiendo de la idea de que el cuerpo habita un contexto (PANIZO, 2011) se analizará cómo, sobre los muertos violentamente, se inscriben y manifiestan relaciones de poder, moralidades, valores y prácticas sociales.

Mientras los accidentes pueden ser pensados como fenómeno asociados al azar (SOUZA MINAYO, 1997), el homicidio y el suicidio se vinculan directamente con riñas, hechos delictivos y conflictos. En este último tipo de casos, se encuentra en disputa el sentido de a quien le cabe la imputación de víctima y victimario, o de 'violento', en cada caso. Como señalan aquellos análisis que reflexionan en torno a la problemática de la violencia (DE HAAN, 2008; RICHES, 1986), lo "violento" es definido por los actores en función de sus criterios morales respecto de lo que ese grupo considera como legítimo e ilegítimo, en determinado contexto (GARRIGA ZUCAL y NOEL, 2010).

La bibliografía que aborda el tópico de la muerte desde las ciencias sociales plantea que, en contraposición con la muerte "normal" (que llegaría en la vejez, sin violencia, en soledad y en un hospital), la muerte violenta es vista como un hecho sorprendente, inesperado, una excepción, un crimen (BAUDRILLARD, 1980; ELÍAS, 1989; THOMAS, 1993; IMBUSCH, MISSE y CARRIÓN, 2011). Sin embargo, en el barrio Santa Ana, la muerte violenta no es algo alejado de la vida cotidiana de las personas. Por el contrario, atraviesa las historias y experiencias de los vivos (PANIZO, 2011; AUYERO y BERTI 2013). Esto abre la pregunta: ¿hay un tratamiento homogéneo de la muerte violenta en diferentes 
contextos y grupos? En función de ella y de la pretendida "homogeneidad" en la actitud de los vivos frente a la muerte, se desprende el interés por buscar heterogeneidades y distintas experiencias en torno a la muerte violenta, que pueden tener lugar entre distintos actores o en distintos contextos.

\section{Campo de estudio: el barrio "Santa Ana", un asentamiento segregado del conurbano bonaerense}

A partir de estos interrogantes previamente mencionados se llevó a cabo, a lo largo del año 2016, un trabajo de investigación etnográfico en el barrio Santa Ana, ubicado al suroeste de la Ciudad Autónoma de Buenos Aires, en el denominado "Conurbano Bonaerense" de la Provincia de Buenos Aires. Este barrio se encuentra a dos horas en transporte público de la Capital Federal, a un kilómetro de distancia de una autopista que oficia de límite entre la Capital Federal y la Provincia de Buenos Aires y que constituye el principal acceso al aeropuerto internacional más importante del país.

Para contextualizar la ubicación y características del barrio, vale aclarar que la provincia de Buenos aires es la jurisdicción más poblada, con mayor densidad y una de las más desiguales del país (SALVIA y VERA, 2016). De acuerdo con un estudio ecológico realizado por la Universidad de Lanús, en los partidos del Gran Buenos Aires o del Conurbano Bonaerense (aquellos que rodean a la Capital Federal), entre los años 1989 y 2006, existe relación entre los índices de violencia y las condiciones de pobreza y desigualdad: "podemos concluir que las crisis económicas están asociadas con el aumento de las tasas de homicidios" (SPINELLI; MACÍAS; DARRAIDOU, 2008). Así, aunque en los últimos años se produjo un ligero descenso (entre 2014 y 2016 la tasa de homicidios pasó a ser de 9,1 a 6,8 por 100.000 habitantes), la Provincia de Buenos Aires continua registrando la mayor cantidad de víctimas de homicidio del país (1.150 en 2016), y su tasa de homicidio se encuentra mayor al promedio nacional (6,8 contra 6), sólo superada por las provincias de Santa Fe, Mendoza Neuquén, Chubut y Formosa (SNIC, 2016).

Según datos oficiales del Ministerio público de la Provincia de Buenos Aires, para el año 2016 la tasa de homicidios en el Conurbano Bonaerense era sensiblemente mayor al resto de los departamentos de la provincia. Esta zona denominada comprende aproximadamente entre el 77 y $78 \%$ de las investigaciones penales preparatorias y el mismo porcentaje de población total de la Provincia. Allí, la tasa de homicidios aumenta a 7,38 cada 100.000 habitantes, contra 4,06 en el resto de la provincia. Por ello es que es factible concluir que el Conurbano Bonaerense presenta mayores niveles de violencia que el resto de la provincia. 
El 86,6\% de las víctimas de homicidios dolosos en la provincia son varones y el $90 \%$ mayores de 18 años (el informe del año 2012 aclara que el 56\% de las victimas tenía entre 18 y 35 años, el $31 \%$ entre 18 y 25 años). En función de la caracterización de los hechos, el informe oficial destaca que el móvil principal es el conflicto interpersonal y que en un 46,9\% existía una relación previa entre víctima e imputado. Este informe también da cuenta de una alta incidencia del uso de armas de fuego en este tipo de hechos: "El principal tipo de arma que se utilizó para consumar los homicidios analizados es el arma de fuego (61,2\%), seguida por el arma blanca $(23,1 \%)$ " (MINISTERIO PÚBLICO DE LA PROVINCIA DE BUENOS AIRES, 2017).

Saliendo de los datos estadísticos de la provincia e ingresando en el barrio Santa Ana, se observan descampados abandonados con basura acumulada y pastizales altos. Entre la autopista y el barrio, se encuentra una zona de monoblocks "tomados", es decir, abandonados y ocupados ilegalmente por personas de bajos recursos. El barrio se creó aproximadamente hace 15 años: es un asentamiento urbano que no tiene servicios de luz y agua, sus viviendas son precarias y no tiene trazado urbano. Las manzanas que organizan el mapa del barrio son delimitadas por pasillos de cemento.

Santa Ana tiene una comisaría, un centro de salud, pero no una escuela. Si bien hay una a siete cuadras, esta privilegia el ingreso de los vecinos de la zona residencial, por los que los niños de Santa Ana asisten a otra escuela a dos kilómetros de distancia, respondiendo a un proceso de segregación social-espacial usualmente vinculado con las formas de marginalización social (BOURDIEU, 2011; WACQUANT, 2001). De la observación y las entrevistas realizadas se desprende que los habitantes presentan altos índices de marginalización social: bajos o nulos ingresos, trabajos informales, viviendas precarias, bajo nivel educativo alcanzado.

El barrio puede ser clasificado como un espacio urbano inseguro por dos motivos. Por un lado, es definido como un lugar peligroso e "inseguro" para los habitantes de los alrededores, debido a la cantidad de hechos delictivos que ocurren. A su vez, en los medios de comunicación se lo define como una zona "en llamas". Se lo describe como un lugar donde cotidianamente se observan tiroteos, robos y muertos. En el 2014, fue noticia al haber sido en sus inmediaciones acribillado un colectivero, lo que derivó en un paro general de micros en pedido de "más seguridad". Por esta percepción respecto a su peligrosidad, el transporte público, reducido a colectivos, no "entra" al barrio, sino que acerca a los transeúntes hasta a dos cuadras. A comienzo del trabajo de campo, el conductor del ómnibus que nos llevó hacia el barrio nos comentó: “Igual no te dejo adentro, ¿Sabes dónde te metes?”. De acuerdo con los 
entrevistados, el personal policial también prefiere no entrar, bajo el lema "yo [ahí] no me meto". Por otro lado, a partir del registro georeferenciado de homicidios en el Conurbano Bonaerense (CSJN, 2012), tanto el municipio como ese barrio en particular, están dentro de las áreas con una mayor concentración espacial de homicidio reportados.

\section{Formas de interpretar las muertes violentas}

Este artículo recupera la tradición de la reconstrucción de escenas como estrategia para ilustrar los procesos y lógicas significativas en los grupos y poblaciones estudiadas (FIGARI, 2008). Esta modalidad de análisis y escritura etnográfica se orienta a la selección e interpretación analítica de procesos, hechos o eventos puntuales, que condensan fenómenos más amplios. En esa línea, se seleccionaron dos escenas en función de su pertinencia para dar cuenta de los procesos sociales y morales que condicionan el registro de datos sobre muertes violentas.

\subsection{Escena 1 - "Al vecino lo sacaron de los pelos"}

La siguiente escena retrata un suceso que, tanto para los propios vecinos del barrio como para los investigadores, resultó excepcional: la muerte violenta de un joven del barrio y el encubrimiento por parte de quienes viven en el asentamiento. El hecho comienza cuando una vecina, Sandra, comenta que a su vecino Pedro lo habían mataron: "Eso todos lo saben. Vivía acá a la vuelta, viste. Cerca de la del kiosco". El problema es que Pedro "andaba de quilombo en quilombo, vendía paco. Y después empezó a consumir y no se rescataba”. Es decir, consumía y vendía drogas, lo que le comenzó a ocasionar problemas de convivencia con la gente de la zona. Según esta vecina, Pedro mantuvo las mismas conductas por un tiempo, alrededor de 3 meses, hasta que en un momento se tornó progresivamente más violento y la gente dejó de tomarlo en "chiste" (como una gracia o travesura) para empezar a verlo con preocupación:

El pibe se empezó a poner medio violento, viste. Y yo me acuerdo que intentó... Intentaba lastimar a las chicas cuando pasaban de noche, ahí en el pasillo. Las manoseaba, no sé. Igual era antes así. Y viste cómo son las cosas acá. Se solucionan de una u otra forma. Mucho tiempo no puedo estar así. Me acuerdo que había estado con dos semanas tirado ahí en el pasillo. Y creo que el problema groso fue cuando se agarró a trompadas con un pibe por no sé qué, cerca de la salita. Y el hermano del pibe se re calentó y lo quiso cagar a trompadas. Y después nada... Se decía que le iban a dar. Y luego bueno una de las noches escuchamos gritos, peleas, se rompían palos, palos, y ya no la volvimos a ver. Ya no estaba más en el barrio. 
Según el testimonio, los problemas que generaba Pedro comenzaron a estar relacionados con acoso y violencia hacia las jóvenes del barrio, lo que culminó con una pelea con otro chico de la zona. Pedro, no sólo generaba problemas, sino que carecía de un espacio de contención emocional familiar o amistoso:

\begin{abstract}
No tenía familia, si era un fisura total. Rancheaba con unos vagos muertos de hambre. Entonces es como que nadie lo defendía y cuando desapareció... Mi hijo hablando con el primo del hijo de los que lo agarraron dijo que lo que le pasó pues... Que se agarró con la gente que no tenía que agarrase y listo. A las creo que a las 3 semanas lo encontraron unos vecinos del fondo. ¿Viste en ese baldío atrás del monoblock? Ahí.
\end{abstract}

La vida de Pedro se caracterizaba por estar solo, relacionarse con "malas juntas", generar problemas asociados a la venta y consumo de drogas, hasta que un día se irrumpe violentamente: a Pedro lo matan y dejan tirado en un baldío. Cuando los vecinos encuentran su cuerpo sin vida, no actúan como lo indican las prácticas consideradas "habituales" frente a un problema, accidente y, más aún, frente hallazgo de un cuerpo sin vida, es decir, llamar al número de emergencias de la policía (el 911). Al contrario, no le dan aviso a la policía. Deciden en conjunto $-\mathrm{y}$ con ello dotan de legitimidad a esa práctica en ese contexto en particular- resolverlo sin acudir a los representantes del Estado. En esa decisión se materializa una primera lógica barrial: ese cuerpo muerto, en ese momento, estaba atravesado por el vínculo de los vecinos del barrio con la policía.

El personal policial entrevistado mencionó que "no se mete" en el barrio y sus problemas, dejando con ello a la zona librada a las normas y reglas producidas y reproducidas por sus habitantes. En este sentido, se puede afirmar que quien tiene el monopolio legítimo del uso de la fuerza del Estado (WEBER, 2014), la fuerza policial, desiste de ejercer sus funciones en este territorio, dejando que lo legítimo e ilegítimo, lo legal e ilegal, no sea definido por el Estado, sino los propios habitantes del barrio (ÁLVAREZ, 2004). Estas fuerzas de seguridad ingresan a estos territorios esporádicamente, en allanamientos ordenados por el poder judicial, procedimientos que duran unas pocas horas y se caracterizan por ser altamente violentos. No patrullan sus zonas, no conocen a sus vecinos, no asisten a llamados de emergencia.

La "lejanía" entre el personal policial y la gente del barrio está fundamentada en dos lógicas. La primera hace referencia a la violencia que las fuerzas policiales ejercen para con estas poblaciones marginales, fundamentalmente con varones jóvenes involucrados en actividades delictivas, y con otros que aunque no lo estén son estigmatizados y 
criminalizados. La segunda implica el rechazo con el que personal policial sería recibido al transitar por estos espacios, siendo blanco de "piedrazos", insultos, entre otras prácticas. Si bien quienes forman las filas policiales suelen provenir de sectores pobres del conurbano bonaerense, manifiestan que al ingresar al mundo policial se "separarían" de los jóvenes conflictivos, dejarían de lado amistades que podrían estar en contacto con el mundo del delito, "pasándose el otro lado", al de las fuerzas encargadas de combatir y reprimir al delito (SIRIMARCO, 2009).

En función de ese vínculo entre policías y habitantes de Santa Ana, es comprensible por qué Sandra decide no reportar el suceso a la policía: "Da para quilombo. ¿Qué van a hacer acá? Ni se meten los cagones. Se quedan afuera. En la salita. Y se van derechito para la autopista. Mejor que no hayan levantado la perdiz". En estas palabras no sólo aparece la decisión de no dar aviso de la muerte, sino el deseo de que la información no circule para que "no se enteren". A su vez, la entrevista con Sandra también muestra que los habitantes del barrio "se manejan" sin la intervención del Estado:

Porque... Yo soy una buena mina. No toco lo ajeno, no me meto con tu marido, laburo de lo que puedo. Pero la verdad es que....La gente... Ese pibe no estaba bien. Nos jodía a todos. No tenía familia. Si no lo desaparecían acá, lo bajaba la cana ${ }^{5}$, o terminaba congelado en una zanja en invierno. Acá no viene el Estado, no viene Scioli ${ }^{6}$, cada tanto los médicos de la salita. Nos tenemos que arreglar entre nosotros los problemas. Cómo una familia, vistes. Y los chicos hicieron eso. Nos defendieron.

De estas palabras surgen varias cuestiones. Por un lado, se destaca la presencia de jóvenes conflictivos a los cuales nadie sabe cómo abordar, incluyendo sus familias, la escuela, los institutos de menores. En ese contexto, aparece la policía como el último eslabón que ejerce violencia sobre estos jóvenes. "Nadie sabe qué hacer con esos chicos" que circulan en el mundo de las drogas, el delito, la desprotección social, la violencia. Por otro lado, se refuerza la idea de que en el barrio todo es gestionado por sus vecinos, incluso la seguridad. "Ellos", los que lo mataron, en definitiva, "los cuidaron". Ese ejercicio de la violencia, en ese momento, en ese lugar y dadas las circunstancias, es legítimo por ese grupo social (GARRIGA ZUCAL, 2015).

Todas estas cuestiones son reafirmadas por Gómez, un policía de la Provincia de Buenos Aires entrevistado:

\footnotetext{
5 Término del lunfardo argentino que se refiere a la policía.

${ }^{6}$ Daniel Scioli era el Gobernador de la Provincia de Buenos Aires en ese entonces.
} 


\begin{abstract}
Sí, escuché que pasó en ese caso en la villa. No me extraña: son lugares que tienen formas como de autoregularse. Cuando estudiaba en la Policía, en una materia, nos decía que en muchos lugares precarios se enseñan estas formas de ajuste de cuenta, de sacar del medio a la gente que causa problemas. Son conductas de pandilla, conductas patológicas. (...). A ese chico que vos decís [el joven asesinado por los vecinos del barrio] lo conocíamos bien: ya había estado adentro por varias cosas menores, digamos. No tuvimos ninguna denuncia cuando desapareció, pero si hicieron referencia desde el centro de salud y algunos de los asistentes sociales, o trabajadores sociales de ahí. La realidad es que como no tuvimos denuncias, ni familiares que vengan con la situación, no hay mucho que podíamos hacer.
\end{abstract}

Con estas palabras, Gómez no se replantea por qué él, como representante del Estado, avala que haya espacios que se autoregulen, ya que el Estado no propone formas de policiamiento comunitarias (RANGUGNI, 2010). Este efectivo policial entiende que la dimensión formal —el "no recibir denuncias"- desliga al personal policial de intervenir, directa o indirectamente, en esos espacios. Pero con sus palabras, Gómez también refuerza la idea de que los familiares y vecinos deciden no hacerlos intervenir y gestionar la seguridad y la justicia por mano propia.

\title{
4.2 ¿Homicidio o suicidio?
}

La primera institución con la que entramos en contacto dentro del barrio fue el centro de salud, ésta fue la vía por la cual ingresamos al barrio, por lo que fue nuestro primer acercamiento a la realidad de Santa Ana. En las primeras entrevistas, los profesionales de la salud hacían referencia a violencias y formas de gestionar las muertes, que serían distintas en comparación con lo que ellos veían en otros barrios del conurbano bonaerense. Un médico, Alberto, en una oportunidad, hizo mención a estas lógicas barriales y cómo ellas incidían en las formas de contabilizar estas muertes.

\footnotetext{
Nosotros sabemos que es importante; que cada caso cuenta en las estadísticas, pero es más complicado. El año pasado encontraron a dos chicos baleados cerca de acá, de la salita: uno más cerca de la autopista, el otro en una especie de baldío. [...]. Viste como es que uno va hablando con la gente y se entera de lo que pasa. Los pibes estaban embromados, venían vendiendo paco en la misma villa, afanaban a los vecinos, estaban siempre fisurados. A la mañana siguiente nos contaban las mujeres [pacientes del servicio] que varios de los vecinos se hartaron de los pibes y los bajaron. Los dejaron en diferentes lados y se cubrieron un poco como para que parezcan suicidios... igual la policía lo sabe. Pero bueno... quedaron como suicidios en las informes [estadísticos de defunción] (Alberto, médico residente).
}

En el fragmento de entrevista, Alberto retrata cómo hay sucesos de los que se entera hablando con vecinos y no por medio de "papeles", que podrían ser producidos por la policía o el sistema de salud. Desde el punto de vista del registro, en esta escena se ponen de relieve 
dos categorías sobre muerte violenta: el suicidio y el homicidio, y cómo los cuerpos pueden ser enmarcados de una u otra manera según el contexto. Si bien los vecinos aseguran que esas muertes fueron homicidios, en los papeles aparecerían como suicidio ¿Qué lógicas hacen posible que esto suceda?

En este caso particular, Alberto considera que existe un pacto de silencio por el cual tanto los vecinos como los funcionarios policiales (únicos referentes estatales mencionados, al margen del médico entrevistado) sabían del asesinato de estos dos jóvenes, pero prefirieron mantenerlo en silencio debido al carácter problemático que representaba para la comunidad. Asociado a estos hechos, este médico describe situaciones y conflictos propios de ese asentamiento, contexto en el cual se enmarcaban esas muertes: el consumo de paco por jóvenes (estupefaciente de baja calidad derivado de la cocaína, sumamente adictivo, consumido por personas de bajos recursos); robo a vecinos; incertidumbre de familias y vecinos sobre qué hacer con estos jóvenes; cansancio de la población sobre los problemas diarios que los aparejan; prácticas habituales de justicia por mano propia. Estos actos violentos, muchos de los cuales terminan en muertes violentas, son significados como altruistas, de solidaridad para con otros vecinos víctimas, son justificados y legitimados al orientarse a dar fin con el flagelo del barrio, asociado a jóvenes problemáticos.

La entrevista con Alberto fue el primer acercamiento sobre este episodio. Luego de sucesivas entrevistas y charlas informales con vecinos del barrio, policías, otros profesionales de la salud y familiares de las dos víctimas, emergieron nuevas aristas que complejizaron nuestra comprensión del episodio. Entre los vecinos de otras zonas del asentamiento y profesionales de instituciones cercanas al barrio (centro de salud y escuelas) circulaba la creencia de que ambos jóvenes habían sido asesinados para "frenar los problemas que estaban trayendo", tal como afirmó una vecina:

\footnotetext{
Yo había escuchado que le robaron a la chica del kiosko, cuando estaba embarazada, y que ahí empezó el problema. ¿¡Cómo le van a robar a una vecina embarazada!? Todos los conocían. El hermano de la chica se calentó, armó un grupito de gente que estaba también cansado de los fisura ${ }^{7}$ estos. Yo estaba de Brain en ese momento. No me puedo imaginar que te quiero afanar estando embarazada.
}

El fragmento de entrevista con la vecina condensa, en gran medida, la creencia de que ambos jóvenes — frente a la rutina de acciones que los vecinos consideraban intolerable e

\footnotetext{
${ }^{7}$ Fisura es un término del lunfardo argentino que, en este contexto, se refiere a alguien con actitud vista como problemática y con consumo problemático de drogas.
} 
incontrolable - fueron asesinados. Nuevamente, las explicaciones varían según la posición que cada vecino ocupa dentro del complejo entramado de relaciones en el asentamiento. Esta cita pone de relieve que los jóvenes habían quebrantado los códigos morales que operaban en el barrio, como robar a los propios vecinos, y en particular a una vecina embarazada.

Esto abre paso a pensar un sistema de "justicia por mano propia", por medio del cual serían resueltos ciertos problemas por modos no "legales y burocráticos", pero no por ello no legítimos en esos contextos. La ausencia de mecanismos de resolución de conflictos por medio de la intervención estatal, la falta de intervención policial, la arraigada creencia de que la justicia estatal "no llega" y, asimismo, la ausencia de un estado benefactor que genere infraestructura y accesibilidad a los jóvenes, generan las condiciones de posibilidad para que un hecho de estas características tuviera lugar. Por ello, este acontecimiento da cuenta de lógicas intrínsecamente barriales que incluyen tanto a representantes del Estado, como a la policía que "sabe y no hace nada" y a los profesionales de la salud; de sistemas de justicia y resolución de conflictos, de gestión de la vida y la muerte, propias de barrios segregados como Santa Ana.

\section{Matar al síntoma. Los jóvenes conflictivos como expresión de la fragmentación del orden barrial}

En uno y otro caso, los chicos catalogados como fisura y descarriados expresan los problemas sociales que aquejan al barrio: marginalidad socio-espacial, falta de intervención estatal - a excepción de dispositivos de emergencia como salitas de salud y policía en el perímetro-, el consumo problemático de drogas, redes de prácticas ilegales vinculadas con las actividades cotidianas en Santa Ana (venta de drogas, diversas formas de abuso y violencia, etc.). Ante la profundidad de las inequidades y la imposibilidad de atacar las causas estructurales de la conflictividad, y ante la ausencia de referentes estatales, sociales e incluso familiares que tuvieran influencia en los jóvenes, se ve como más sencillo "atacar al síntoma que curar la enfermedad".

Esta circunstancia da cuenta de un mecanismo social más profundo que la inequidad y conflictividad social del barrio. Revela que en el barrio se sustentan una serie de valores en torno a la convivencia pacífica y que estos fueron denigrados por los jóvenes. Las tropearías cometidas por los jóvenes los oponen a una noción de reciprocidad comunal y, de este modo, se convierten en enemigos de la comunidad en el sentido schmittiano (SCHMITT, 2005; ÁLVAREZ, 2004). Así, el respeto por las reglas implícitas de convivencia pacífica delimitan un nosotros, y configuran un otros-enemigo por parte de aquellos que no lo hacen. La 
extranjería de estos jóvenes, justificaría el asesinato desde la perspectiva de los actores. La delimitación de un nosotros y un ellos construido como problema para la comunidad, posibilitó el curso de acción tomado, permitiría ante los ojos del barrio sostener una versión de inocencia e impunidad, ante el acometido de un homicidio.

Mientras en la primera escena relatada el caso no llegó a las autoridades, permitiendo la omisión de denuncia y el registro estadístico, en la segunda escena la continuidad del pacto de silencio se da mediante una suerte de complicidad del personal policial que — pese a cumplir su obligación de registrar el hecho-toma distancia en lo discursivo, pero actúa de forma connivente al "simplificar el proceso burocrático" y rotular el caso como muerte autoinfligida.

Al consultarle a Germán, un policía que solía trabajar en el barrio en el momento en el que encontraron a los dos jóvenes sobre el caso, señaló:

Yo sé que podría haber sido un homicidio. Hay muchos casos como estos, con chicos descarriados en estos barrios. Pero, a ver, no había pruebas de nada. Y además siempre es más complicado empezar una investigación de homicidio que de suicidio. A veces, cuando uno conoce el lugar y sabe que no va a pasar nada, que no se va a encontrar a nadie, es más fácil simplificar el proceso burocrático, ¿me entendés? Además le ahorrás sufrimiento a la gente. Y no malgastas el dinero del Estado [Cursivas agregadas].

Frente a los rumores y teorías sobre el asentamiento y sobre algunas de las instituciones vinculadas con la vida cotidiana del barrio, en los policías se mantiene una postura distante sobre el hecho. No generaron una investigación profunda sobre el caso y justifican esto por la creencia de que no es "necesario" buscar de más. Esta omisión es respaldada mediante dos argumentos: por un lado, no "malgastar el dinero del Estado" en algo que no valdría la pena; por otro lado, conocer la dinámica del lugar, que tendría lógicas propias que se deben respetar. Respecto a estas lógicas barriales, Sebastián, otro policía de la comisaría cercana al barrio, afirma:

Son excepciones. No es algo frecuente, claro. No se puede negar que existan esos casos. Las... villas [duda al usar el término] tienen una forma de obrar, la gente se maneja con ciertos códigos... peligrosos. Y no estoy de acuerdo con los que dicen que 'no tiene códigos'. Tienen códigos, pero son códigos sociopáticos, delictivos, ¿entendés?

Por un lado, ante el Estado de Excepción que caracteriza a estos espacios (AGAMBEN, 2005), el policía se reconoce extraño a este lugar y lo ubica fuera del ámbito de 
la legalidad que él ejerce. Así plantea que sería mejor no seguir buscando pruebas de homicidio, ya que es más difícil que cerrar el caso como un suicidio.

Por otro lado, el policía destaca una premisa circulante dentro de muchos de los policías entrevistados: es conveniente propiciar el ahorro de gastos en recursos estatales y de sufrimiento a la gente. No obstante, tras a una primera aproximación a esta creencia, la categoría de "gente" pierde su aparente universalidad. Para los policías, "gente" refiere a un conjunto de sujetos más restringido que los ciudadanos: "gente" hace referencia a quienes no infringen la ley o, al menos, quienes no establecen una relación antagónica con la policía.

Ante la dificultad de pensar la muerte de un 'descarriado' como un hecho delictivo que merece sanción, o al joven descarriado como una víctima, y ubicar al barrio como un lugar particular, por fuera de las lógicas de legalidad que debe impartir, el policía concluye que no es necesario investigar una muerte anunciada sobre la que nadie pedirá explicaciones adicionales.

\section{Inhibición social de la condición de victima}

En la segunda escena ambos jóvenes tenían allegados en el barrio. Sus círculos más cercanos — familiares directos, amigos y algunos vecinos que tenían trato con ellos-, habían desarrollado diferentes explicaciones e hipótesis sobre las muertes: ajuste de cuentas entre bandas, venganza e intento de robo fueron algunas de las explicaciones que se habían estructurado - con diferente nivel de coherencia y extensión dentro de estos círculos- para explicar qué les había pasado a ambos jóvenes. A su vez, dentro de estos grupos, las explicaciones variaban según las particularidades que los vínculos traían a los relatos: disputas entre los dos jóvenes, rivalidades con otras bandas y peleas por una misma mujer, eran algunos de los componentes que se priorizaban y esgrimían para moldear explicaciones.

Todas las argumentaciones de los allegados esbozaban algún grado de responsabilidad de las propias víctimas sobre este resultado final, que incluso lo volvían esperable. Es decir, existía un grado de sospecha de que los jóvenes estaban en un ámbito delictivo, lo cual tornó que las lesiones, violencias y muertes fueran tenidas en cuenta como eventos potenciales. Por el contrario, el carácter no esperable de la muerte de otros vecinos, los vecinos "decentes no vinculados con actividades delictivas", los tornaban potenciales víctimas.

Los vecinos "ajusticiadores" no sólo mataron sino que tiraron los cuerpos en un baldío y cerca de la autopista. Recordemos que, para Hertz (1990) la intensidad de las emociones provocadas por el deceso depende de los valores sociales de los difuntos: "la muerte de un extranjero, de un esclavo o un niño pasará casi desapercibida, sin provocar emoción ni dar 
lugar a rito alguno" (Hertz, 1990: 88). Los modos de honrar y recordar a los muertos da cuenta de la jerarquía social de estos cuerpos (Ariés, 1984; Baudrillard, 1980; Thomas, 1993). En los casos de análisis, los tres difuntos se caracterizaban por la negatividad: asociados al consumo de drogas, a la delincuencia, al abandono, a la agresividad, cobardes por increpar a las mujeres del barrio. No eran considerados miembros preciados de la comunidad. Por el contrario, los agravios cometidos los acercaban más a la condición de extranjero o enemigo (SCHMITT, 1932) de los habitantes del asentamiento.

Su condición de 'otros que molestan' es palpable a tal punto que los mismos allegados de las víctimas fatales sostienen algún tipo de justificación por el resultado fatal y evitan cualquier tipo de reclamo por su esclarecimiento. Exigirlo no sería reconocido como un acto de justicia, ante la actitud vergonzante de los difuntos, a la vez que generaría tensión con el resto de los habitantes, poniendo en peligro incluso su pertenencia a la comunidad. En consecuencia, deben soportar el acto vejatorio que implica el arrebato de la vida mediante la violencia y su arrojo en un baldío. De este modo quedan excluidos de la posibilidad de duelo y se borra su condición humana (BUTLER; 2006). Se produce el fenómeno contrario en torno a la construcción de una víctima social (KESSLER, 2015), por el cual la muerte de un miembro de la comunidad desencadena un proceso de reconocimiento del cuerpo muerto como víctima de un hecho injusto que merece ser esclarecido y motiva el reclamo político de búsqueda de justicia (PITA, 2010).

\section{Reflexiones finales}

Las dos escenas retratadas en Santa Ana permiten abordar una serie de puntos en torno a las violencias, el registro de datos oficiales y, asimismo, la potencialidad del trabajo etnográfico. Se destacan, por un lado, aspectos generales vinculados con el subregistro y omisión de datos que trascienden el escenario argentino y, por el otro lado, aspectos de nivel local relacionados con las dinámicas propias del espacio social que hemos abordado empíricamente.

En un nivel más general, se ratificó —en el contexto local- lo que otras investigaciones afirman sobre la producción de datos sobre violencias (NOYMER, PENNER Y SAPERSTEIN, 2011; MENÉNDEZ, 2012; SCHEPER-HUGHES, 2000): los contextos socio-culturales y los repertorios morales de los actores sociales "en territorio" moldean la clasificación de las muertes. Status, representaciones sociales, dinámicas grupales y formas de vulnerabilidad están imbricados en el proceso de delimitación sobre qué se clasifica y qué no, 
cómo y quién tiene la legitimidad de hacerlo. Lejos de ser un planteo menor, esto abre la discusión sobre la objetividad de los datos estadísticos y, aún más, permite relativizar la centralidad de los indicadores sobre violencias en la descripción general de la situación de las poblaciones. En este sentido, estos casos muestran cómo el registro de los homicidios no depende solamente de la "norma" escrita (certificado estadístico de defunción, procedimientos judiciales, etc.), sino de una realidad interaccional. No obstante, queda pendiente la discusión acerca de si los problemas de registro estadístico se acentúan o concentran en espacios relegados económicamente $\mathrm{o}$ en los cuales se superponen diferentes procesos de vulnerabilización, como lo demuestran otros estudios sobre mortalidad (NOYMER, PENNER Y SAPERSTEIN, 2011; MINSAL, 2008).

Los eventos y procesos en Santa Ana demuestran que el registro de datos estadísticos es, necesariamente, multidimensional. El tipo de muertes está mediada por un conjunto de interpretaciones que dependen del entramado de actores que las clasificó y esto abre diversos interrogantes: ¿qué tan representativos fueron estos casos? ¿Hubo un interés por que las muertes fuesen clasificadas como suicidios? ¿Qué relevancia le dan los vecinos a las clasificaciones estatales sobre la muerte? ¿Qué relevancia le dan los policías a las explicaciones y disputas de sentido dentro del barrio?

En un segundo nivel más anclado en la realidad del Conurbano Bonaerense, las escenas detalladas permiten desentrañar una particularidad socio-antropológica en este contexto. Las cadenas de violencia que se reproducen en los barrios marginalizados (AUYERO Y BERTI, 2013), así como los tensos, pero cercanos vínculos entre policías y población marginalizada (GALVANI M., 2016) se entremezclan en la realidad del barrio, generando condiciones de posibilidad para la forma de registro de los homicidios. Principalmente, este escenario sienta las bases para los márgenes de maniobra de los actores allí presentes: dinámicas de venganza internas del barrio, la autonomía relativa de los policías frente a las normas oficiales, la falta de intervención de otros actores estatales, etc.

A su vez, aquí se mostró cómo operan la falta de condiciones materiales y simbólicas de "producción" de víctimas, ya abordada en profundidad por la literatura antropológica (PITA, 2010; GUTIERREZ, 2011; DAMMERT y SALAZAR, 2009; KESSLER y GAYOL, 2015). Tanto para los vecinos del barrio, como para los diferentes actores estatales que reticularmente trabajan en el barrio (policías, médicos, trabajadores sociales, etc.), estas muertes retratadas no pueden ser (o difícilmente lo sean) consideradas víctimas visibilizadas y registradas. 
Por último, el trabajo de campo y la discusión sobre el ("correcto") registro de estadísticas vitales conlleva una discusión ética que, si bien no se ha abordado en este artículo, no deja de ser pertinente y necesaria de ser mencionada. ¿Qué posturas entran en tensión frente a los hallazgos de esta etnografía? El registro incorrecto de datos oficiales, el homicidio silenciado de jóvenes, la falta de intervención de las autoridades estatales y la "impunidad" frente a hechos de violencia tornan a este escenario en una situación paradigmática del campo de estudios de las violencias. Por un lado, se visibiliza la opacidad de lo usualmente considerado fidedigno. Por otro lado, refuerza la pertinencia de las ciencias sociales en la investigación empírica y reflexiva en el estudio del fenómeno complejo de la violencia, como forma de desnaturalizar y visibilizar los procesos sociales que la sustentan.

\section{Referencias bibliográficas}

ALONSO, W. STARR, P. Politics of numbers The Politics of Numbers. Population of the United States in the 1980s. A Census Monograph Series. Nueva York: Russell Sage Foundation, 1987. 496 p.

ALVAREZ, S. Leviatan y Sus Lobos: Violencia y Poder En Una Comunidad de Los Andes Colombianos. Buenos Aires: Antropofagia, 2004. 223 p.

AUYERO, J. BERTI, M.F. Violencia en los márgenes: una maestra y un sociólogo en el conurbano bonaerense. Buenos Aires: Katz, 2013. 174 p.

AZEVEDO Y CIFALI, A. C. Seguridad Pública, Política Criminal y Penalidad en Brasil durante los Gobiernos Lula y Dilma (2003-2014). Cambios y Continuidades. En SOZZO, M. (Comp.). Postneoliberalismo y penalidad en América del sur. Ciudad Autónoma de Buenos Aires: CLACSO, 2016. p. 29-94.

BALL, P.; REED H. M. El registro y la medición de la criminalidad. El problema de los datos faltantes y el uso de la ciencia para producir estimaciones en relación con el homicidio en Colombia, demostrado a partir de un ejemplo: el departamento de Antioquia (2003-2011). Revista Criminalidad, v. 58, n.1, 2016.

BOURDIEU, P. El sentido práctico. Buenos Aires: Siglo XXI Editores, 2010. 456 p.

BERMÚDEZ, N. "Y los muertos no mueren". Una etnografía sobre clasificaciones, valores morales y prácticas en torno a muertes violentas (Córdoba, Argentina). Barcelona: Editorial Académica Española, 2011. 211 p.

BOURDIEU, P. La reproducción. Elementos para una teoría de la enseñanza. Barcelona: Editorial Laia, 1979. 285 p.

BRICEÑO-LEÓN, R. La Comprensión de los Homicidios en América Latina: ¿Pobreza o Institucionalidad? Ciência \& Saúde Coletiva, v. 17, n. 12, 2012, p. 3159-3170. 
BUITRAGO, J. R.; NORZA, E. Registros de la criminalidad en Colombia y actividad operativa de la Policía Nacional durante el año 2015. Revista Criminalidad, v. 58, 2016. p. 920.

BUTLER, J. Vida precaria. El poder del duelo y la violencia. Barcelona: Paidós, 2016. 192 p.

BUVINIC, M.; MORRISON, A. Notas técnicas: prevención de la violencia. Nota Técnica No.2. Washington DC. BID, 1999.

CASTRO, M. S., MONTEIRO DE ASSUNÇÃO, R. M; DURANTE, M. O. Comparação de dados sobre homicídios entre dois sistemas Sistema de Informações, Minas Gerais. Revista de Saude Publica, v. 37, n. 2, 2003. p. 168-176.

CORTE SUPREMA DE JUSTICIA DE LA NACIÓN (CSJN). Homicidios dolosos 2012 Conurbano Bonaerense, Buenos Aires, s/f. Disponible en: http://www.csjn.gov.ar/investigaciones/ Acceso: 30 de noviembre 2017.

DAMMERT L., SALAZAR. F. ¿Duros con el delito?: populismo e inseguridad en América Latina. Santiago de Chile: FLACSO, 2009.

DAMMERT L. y ZÚÑIGA L. Seguridad y violencia: desafíos para la ciudadanía, Santiago, Chile: FLACSO, 2007. 277 p.

DÁVILA-CERVANTES C. A.; PARDO-MONTAÑO A. M.. Magnitud y tendencia de la mortalidad por homicidios en Colombia y México, 2000 - 2011, Facultad Latinoamericana de Ciencias Sociales sede México (Flacso). Distrito Federal, México. Disponible en: https://scielosp.org/scielo.php?pid=S1020-49892014000600002\&script=sci_arttext._Acceso: 30 de noviembre 2017.

DIRK, R. C. Homicídio doloso no Estado do Rio de Janeiro: uma análise sobre os registros de ocorrência da Polícia Civil. Rio de Janeiro, Brasil: Dissertação (Mestrado em Estudos Populacionais e Pesquisa Social), Escola Nacional de Ciências Estatísticas, 2007.

FIGARI, C. Heterosexualidades masculinas flexibles (pp. 97-122). En Pecheny, M.; Figari, C. y Jones, D. Todo sexo es político. Estudios de sexualidad en Argentina. Buenos Aires: Libros del Zorzal, 2008.

FERNÁNDEZ, A.M. Femicidios: la ferocidad del patriarcado. Revista Nomadías, n. 16, p. 47-73, 2012.

FLEITAS ORTIZ, R. Homicidios y suicidios en Argentina Alcances y evolución de 1997 al 2012, Documento de Trabajo, Buenos Aires: Asociación para las políticas públicas, Septiembre 2014.

GALVANI, I. Cuestión de "cintura”. Formas de obedecer y desobedecer en el personal subalterno del Servicio Penitenciario Bonaerense. En Frederic, S., Galvani, M., Garriga ZUCAL, J., RENOLDI, B. (editores), De Armas Llevar. Estudios socioantropológicos sobre los quehaceres de policías y de las fuerzas de seguridad, Buenos Aires: EPC Ediciones, 2013. 115-146 p. 
GALVANI, M. Cómo se construye un policía. La Federal desde adentro. Buenos Aires: Siglo XXI Editores, 2016. 240 p.

GEERTZ, C. El antropólogo como autor, Barcelona: Paidos, 1989.

GOVEA BASCH. Lo que todavía debemos mejorar de las estadísticas vitales, Población de Buenos Aires. Año 7, $\mathrm{n}^{\circ}$ 11, 2010. Disponible en: http://www.redalyc.org/html/740/74012783007/ Acceso: 30 de noviembre 2017.

GRIZE, J. B. Psicologia genética e lógica. En: BANKS-LEITE, L. (Org.). Percursos piagetianos. Sao Paulo: Cortez. 1997. p. 63-76.

GUBER, R. La etnografía: método, campo y reflexividad, Buenos Aires: Universidad de La Plata, 2005.

GUTIERREZ, M. Populismo punitivo y justicia expresiva, Revista de derecho Penal y Criminología, ISSN 0034-7914, Nº 3, 2011.

KESSLER G.; GAYOL, S. Muerte, política y sociedad, Ciudad Autónoma de. Buenos Aires: Edhasa, 2015.

MALDONADO ARANA, S. Desafíos etnográficos en el estudio de la violencia. Experiencias de una investigación, Misiones Argentina, Avá no.22, abr. 2013.

MATUS, C. Política, planificación y gobierno. Caracas: Fundación Altadir. 1992.

MAZZEO, V. Serie de diagnósticos sobre la calidad de la información de las estadísticas vitales ocurridas y registradas en la Ciudad de Buenos Aires en el período 1993-2008, Buenos Aires, 2008.

MAZZEO, V., “¿Qué debemos mejorar en el registro de las estadísticas vitales?”, en Revista Población de Buenos Aires, año 2, n 2, Buenos Aires, DGEyC, septiembre, 2005.

MENENDEZ, E. Metodología cualitativa: varios problemas y reiteradas omisiones, Index Enferm vol.21 no.1-2 Granada ene./jun. 2012.

MÉXICO EVALÚA. Índice de víctimas visibles e invisibles de delitos graves El daño que no se conoce, Agosto 2011.

MÍGUEZ D.; ISLA A. Entre la inseguridad y el temor: Instantáneas de la sociedad actual. Buenos Aires: Paidós, 2010.

MINISTERIO DE SEGURIDAD DE LA NACIÓN REPÚBLICA ARGENTINA, Informe del Sistema Nacional de Información Criminal (SNIC), Año 2016, Junio 2017, Disponible en: https://estadisticascriminales.minseg.gob.ar/reports/Informe\%20SNIC\%202016.pdf Acceso: 30 de noviembre 2017.

MINSAL/OPS. Omisión de registro de nacimientos y muertes infantiles. Magnitud desigualdades y causas. Buenos Aires, MINSAL/OPS. 2008. 198 p. 
MIRANDA, A. P. M.; PITA, M. V. O que as cifras cifram? Reflexões comparativas sobre as políticas de produção de registros estatísticos criminais sobre mortes violentas nas áreas metropolitanas do Rio de Janeiro e de Buenos Aires. In: KANT DE LIMA, R.; PIRES, L.; EILBAUM, L. (orgs.). Burocracias, direitos e conflitos: pesquisas comparadas em Antropologia do Direito. Rio de Janeiro: Garamond. 2011. p. 175-202.

MIRANDA, A. P. M.; DIRK, R. Análise da construção de registros estatísticos policiais no Estado do Rio de Janeiro. En: KANT DE LIMA, R.; EILBAUM, L.; PIRES, L. (orgs.). Conflitos, direitos e moralidades em perspectiva comparada. Rio de Janeiro: Garamond, 2010.

NOYMER, A.; PENNER, A.; SAPERSTEIN, A., Cause of Death Affects Racial Classification on Death Certificates, Plos One, Volume 6. 2011. p. 1-3

OTERO, H. Estadística y Nación, Buenos Aires: Prometeo, 2007.

PITA M. Formas de morir y formas de vivir: el activismo contra la violencia policial. Buenos Aires: Del Puerto/CELS, 2010.

PROCURADURÍA DE VIOLENCIA INSTITUCIONAL (PROCUVIN). Fallecimientos en contextos de encierro Análisis de muertes en cárceles del Servicio Penitenciario Federal (2010-2014), Área de Registro y Bases de Datos Procuraduría de Violencia Institucional, Ministerio Público Fiscal de la Nación, s/f. Disponible en: https://www.mpf.gob.ar/procuvin/files/2015/09/Informe-sobre-muertes-en-contexto-deencierro-2010-2014-Fina.pdf Acceso: 30 de noviembre 2017.

RAMÍREZ-HITA, S. La contribución del método etnográfico al registro del dato epidemiológico. Epidemiología sociocultural indígena quechua de la ciudad de Potosí, Salud colectiva v.5 n.1 Lanús ene./abr. 2009.

RICHES, D. The Anthropology of violence, Blackwell Publishers, 1986.

SALVIA, A. (Coordinador del estudio), Vera J., (Investigadora responsable), Pobreza y desigualdad por ingresos en la argentina urbana 2010-2015. Tiempos de balance, Buenos Aires: Observatorio de la Deuda Social Argentina, Universidad Católica Argentina, 2016.

SARRABAYROUSE OLIVEIRA M. J. Poder judicial y dictadura. El caso de la morgue judicial, Cuaderno $N^{o}$ 4: Memoria y dictadura. Defensoría del Pueblo de la Ciudad de Buenos Aires/. Facultad de Filosofía Letras, Universidad de Buenos Aires, 2003.

SCHEPER-HUGHES, N. Demografía sin números. El contexto económico y cultural de la mortalidad Infantil en Brasil. En: Viola, A. (comp.). Antropología del desarrollo. Teorías y estudios etnográficos en América Latina. Barcelona, Paidós: 2000.

SCHMITT C. El concepto de lo político, Buenos Aires: Alianza Editorial, 1936. 
SOUZA MINAYO, M.C. Violência e saúde como um campo interdisciplinar e de ação coletiva. EnHistória, Ciências, Saúde-Manguinhos, Hist. cienc. saude-Manguinhos, vol.4, n.3, 1997.

SPINELLI, H.; MACÍAS, G.; DARRAIDOU, V. Procesos macroeconómicos y homicidios. Un estudio ecológico en los partidos del Gran Buenos Aires (Argentina) entre los años 1989 y 2006. Salud Colectiva, v. 4, n. 3, p. 283-299, 2008.

SPINELLI, H.; ALAZRAQUI, M; MACÍAS, G. Muertes violentas en la Ciudad Autónoma de Buenos Aires. Una mirada desde el sector salud. Buenos Aires: Organización Panamericana de la Salud, 2005. 96 p.

WACQUANT, L. Parias urbanos: marginalidad en la ciudad a comienzos del milenio. Buenos Aires: Manantial, 2001. 208 p.

YUNES, J.; ZUBAREW, T. Mortalidad por causas violentas en adolescentes y jóvenes: un desafío para la región de las Américas. Revista Brasileira de Epidemiologia, v. 2, n. 3, p. 102 $171,1999$. 